\title{
T-cell vaccination leads to suppression of intrapancreatic Th17 cells through Stat3-mediated ROR $\gamma$ t inhibition in autoimmune diabetes
}

Min Wang ${ }^{1}$, Liu Yang ${ }^{1}$, Xiaoyan Sheng ${ }^{1}$, Weilei Chen ${ }^{1}$, Haiqing Tang ${ }^{1}$, Hongguang Sheng ${ }^{2}$, Beili Xi Ying Qin Zang ${ }^{1}$

${ }^{1}$ Institute for Nutritional Sciences, Shanghai Institutes for Biological Sciences, Graduate School of CAS, Chinese Academy of Sciences, Shanghai 200031, China; ${ }^{2} X u h u i$ Central Hospital, 966 M. Huaihai Road, Shanghai 200031, China

Immunization with inactivated autoreactive $T$ cells is an effective therapeutic approach to ameliorating autoimmune diseases, while the underlying mechanisms that regulate autoreactive $T$ cells are not completely understood. This study tested the hypothesis that T-cell vaccination (TCV) inhibits autoimmune diabetes in mice through the suppression of Th17 cells. The results showed that TCV treatment decreased hyperglycemia in type 1 diabetes (T1D) induced by multiple low-dose streptozotocin (MLD-STZ) as compared with the controls, preserved the number of healthy pancreatic islets and increased the production of insulin in the islets. Further study revealed that TCV significantly decreased the production of both interleukin (IL)-17 and IL-23 in intrapancreatic infiltrating lymphocytes (IPL) through marked inhibition of mRNA level of retinoic acid-related orphan receptor $\gamma t(R O R \gamma t)$ and signal transducer and activator of transcription 3 (Stat3) phosphorylation. The role of TCVinduced Th17 suppression was further validated in adoptive transfer experiments with polarized Th17 cells in subdiabetogenic mice, which was similar to the effect of anti-IL-17 antibody treatment. Collectively our study shows that intrapancreatic Th17 cell suppression and healthy islet preservation play an important role in the treatment of T1D by TCV.

Keywords: type 1 diabetes; T-cell vaccination; Th17; intrapanceatic infiltrating lymphocytes Cell Research (2011) 21:1358-1369. doi:10.1038/cr.2011.74; published online 26 April 2011

\section{Introduction}

Type 1 diabetes (T1D) is an autoimmune disease resulted from selective destruction of the insulin-producing $\beta$ cells in the pancreatic islets, and is primarily a T-cellmediated autoimmune disease directed against one or more $\beta$-cell autoantigens. Hyperglycemia will occur and insulin supplement will be needed after most of the

Correspondence: Ying Qin Zang ${ }^{\mathrm{a}}$, Beili Xi

${ }^{a}$ E-mail: yqin@sibs.ac.cn

bE-mail: xi.beili@hotmail.com

Abbreviations: GAD (glutamic acid decarboxylase); IPL (intrapanceatic infiltrating lymphocytes); MLD (multiple low dose); STZ (streptozotocin); SMCs (spleen mononuclear cells); TCV (T-cell vaccination); T1D (type 1 diabetes)

Received 29 August 2010; revised 14 December 2010; accepted 6 January 2011; published online 26 April 2011 $\beta$ cells have been eradicated [1]. At present, treatment of T1D mainly focuses on restraining the endocrine/ metabolic disease with insulin, and anti-hypertensive and lipid-lowering agents. The major weakness of this therapeutic approach is that it has no effect on the autoimmune process, the key element in the pathogenesis of diabetes. It has been reported that $\mathrm{T}$-cell vaccination (TCV) offers some advantages over the treatment with insulin or insulin replacement [2]. The TCV approach targets the immune system by inhibiting the progression of the inflammatory process. This immune-modulating approach attempts to not only cure but also prevent the onset of T1D, and thus avoid the consequences of the endocrine disease. The rationale and practice of TCV against autoimmune diseases are similar to classical vaccination against infectious diseases. Although the precise mechanism remains to be elucidated, various studies indicate that TCV elicits its function by activating Th1- 
type regulatory cells that can block the Th1 autoimmune $\mathrm{T}$ cells and switch the autoimmune response to a Th2 phenotype [3, 4]. In other works, Th1 regulators, such as Tregs, convert a Th1 autoimmune attack into a suppressive Th2 response $[4,5]$. Th1 cells produce interferon- $\gamma$ (INF- $\gamma$ ) and regulate cellular immunity, whereas Th2 cells produce interleukin-4 (IL-4), IL-5, and IL-13 and mediate humoral immunity and allergic responses. Th1/ Th2-cell subsets have been extensively studied and used to explain T-cell functions in immune responses and diseases. Th2 used to be considered protective in organ-specific autoimmune diseases, while Th1 cells are inflammatory and can be pathogenic. However, several studies reported that deficiency in IFN- $\gamma$ or IL-12 does not decrease but increases the onset and severity of disease in mouse models of autoimmunity [6-12]. It has been increasingly recognized that in many of the mouse autoimmune disease models that have been attributed to Th1 cells, disease severity is actually increased in the absence of these cells. For example, experimental autoimmune encephalomyelitis (EAE) and collagen-induced arthritis are exacerbated in the absence of IFN- $\gamma$, IFN- $\gamma$-R, signal transducer and activator of transcription 1 (Stat1), or IL12 p35 [13, 14]. However, mice lacking the IL-12 p40 subunit, which is shared with IL-23, are resistant to those autoimmune diseases $[7,11]$. This paradox can be solved by the characterization of a third subset of T-helper cells, Th17 cells [13-15]. These cells produce IL-17, IL17F, IL-22, and IL-21, and mediate tissue inflammation. There is growing evidence indicating that Th17 cells are pathogenic in several autoimmune disease models, such as EAE and collage-induced arthritis [7, 15-17]. At present, there is limited information available on the role of Th17 cells in T1D mellitus.

STZ is a D-glucopyranose derivative of $N$-methyl- $N$ nitrosourea endowed with potent alkylating properties [18] and also acts as a reactive oxygen species-generating agent [19]. While at high doses this agent has diabetogenic potential due to its capacity to selectively promote death of insulin-producing $\beta$ cells by apoptosis or necrosis, at low doses, STZ generates hydrogen peroxide [20] and induces expression of the glutamic acid decarboxylase autoantigen [21]. Glutamic acid decarboxylase (GAD) is a strong trigger of $\beta$-cell-specific autoimmunity, both in humans and experimental models of diabetes [22-24]. In this study, we used multiple low doses (MLD) of streptozotocin (STZ)-induced type 1 diabetes as our animal model and demonstrated the involvement of Th17 cells in the pathogenesis of T1D. We further investigated the mechanisms underlying the therapeutic effect of TCV on STZ-induced T1D in association with its influence on Th17 cells.

\section{Results}

TCV ameliorated the development of autoimmune diabetes in MLD-STZ diabetic mice

To investigate the effect of TCV in experimental diabetes, we used a diabetogenic regimen of MLD-STZ treatment, which could induce sustained and progressive hyperglycemia and insulitis in male C57BL/6 mice [20]. T cells specific to GAD65 p206-220 (TCV) and control TCV specific for hen egg lysozyme (HEL) p1125 (TCV-HEL) were inoculated subcutaneously to MLDSTZ mice on day $0,7,14$ with control mice receiving the carrier saline only. All mice developed progressive and sustained hyperglycemia in STZ group and TCV-HEL group. However, TCV significantly decreased the blood glucose level 14 days after the vaccine administration and inhibited diabetogenesis compared with the STZ and TCV-HEL groups (Figure 1A and 1B). In all, $72.7 \%$ of the mice treated with TCV were protected against diabetes throughout the study period and only one mouse developed moderate hyperglycemia and another two mice had a slight increase of blood glucose level. TCV treatment not only halted the decrease in the serum insulin level (Figure 1C) but also increased in situ production of insulin as shown by immunohistochemistry using the anti-insulin antibody on day 21 of the TCV treatment (Figure 1D). Using hematoxylin and eosin staining, we counted a total of 100 islets on paraffin-embedded pancreatic tissue sections and found that the TCV group retained 50 healthy islets, whereas untreated mice had only 10 healthy islets (Figure 1E). Insulitis was significantly ameliorated in the TCV-treated mice compared with the untreated controls. The criteria for insulitis scoring are illustrated in Figure 1F. The numbers of islets with severe and mild intrainsulitis were reduced in the TCV-treated mice vs non-treated ones ( $6 \%$ and $13 \%$ vs $42 \%$ and $34 \%)$. The results suggest that TCV-treatment decreased hyperglycemia in T1D induced by MLD-STZ, preserved the number of healthy pancreatic islets and increased the production of insulin in the islets.

Cytokine profile of intrapancreatic infiltrating lymphocytes and spleen mononuclear cells (SMCs)

The role of inflammatory cytokines in diabetes and its complications is well established [25]. These molecules can be used as important markers to evaluate the onset, progression, and treatment of diabetes [26]. We measured cytokine profile in culture supernatants of intrapancreatic infiltrating cells and SMCs from TCV-treated, nontreated MLD-STZ diabetic mice, and normal C57BL/6 mice. As shown in Figure 2, TCV treatment reduced the levels of INF- $\gamma$ and TNF- $\alpha$ in the culture supernatant of 

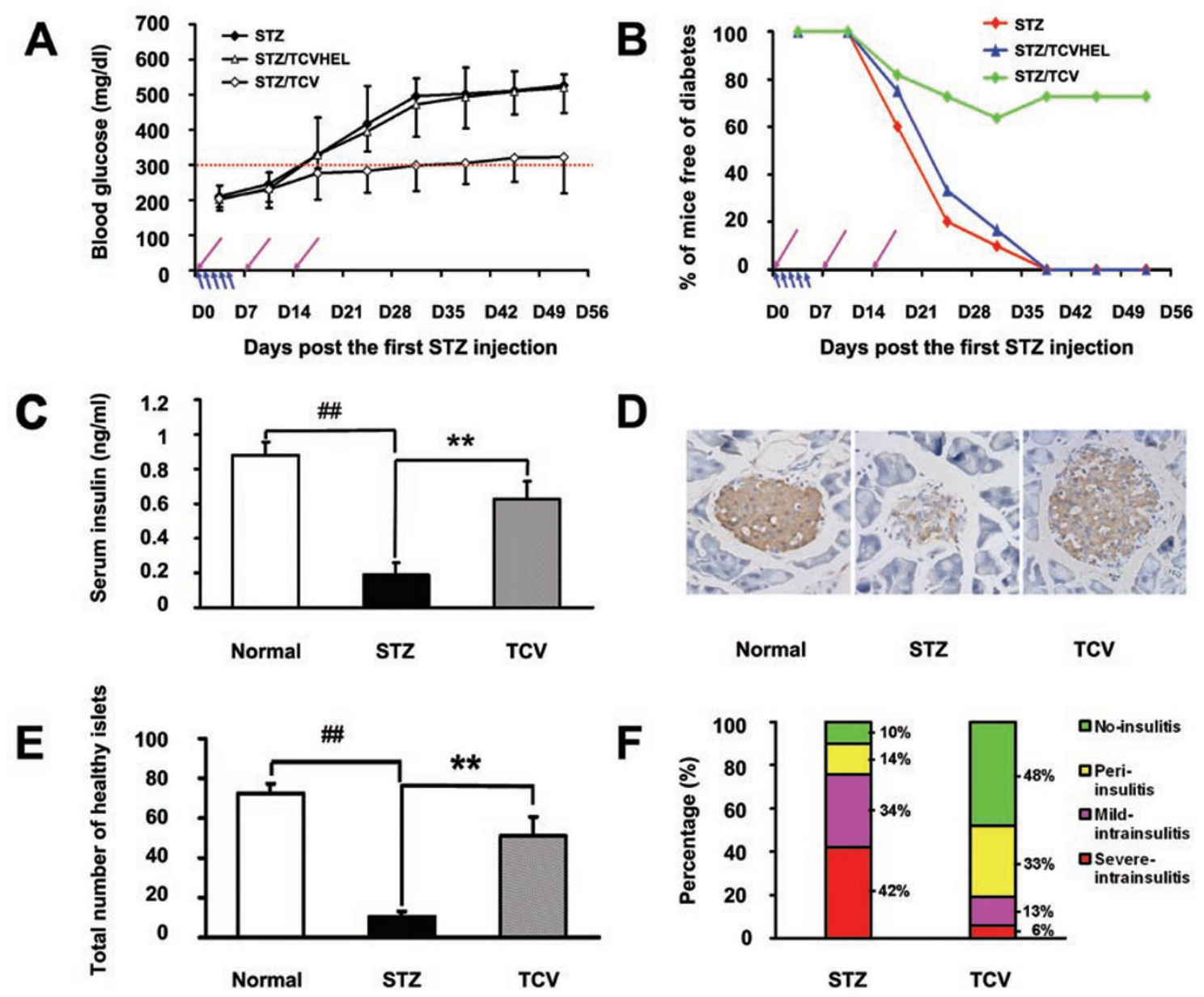

Figure $1 \mathrm{TCV}$ improved the development of autoimmune diabetes in MLD-STZ diabetic mice. (A) Blood glucose levels of TCV-treated mice (STZ/TCV), TCVHEL-treated mice (STZ/TCVHEL) and controls (STZ) are shown from day 0 to day 56 . (B) Percentage of mice free of diabetes through the whole experiment. Purple arrows indicate the days when TCV and TCVHEL were given, and blue arrows indicate all five STZ injections from day 0 to day 4. (C) Serum insulin levels in TCV-treated mice (TCV) and controls (STZ) on day 21. (D) In situ production of insulin in healthy mice (normal), MLD-STZ diabetic mice (STZ) and TCV-treated MLD-STZ diabetic mice (TCV) on day 21. (E) Total islets per pancreas as determined by hematoxylin and eosin staining from three groups of mice on day 21. (F) Islets from normal, TCV-treated and non-treated mice were scored as described in Materials and Methods, and the percentages represent the number of islets of a given score over the total number of islets from (E). ${ }^{\#} P<0.01$ and ${ }^{* *} P<0.01$ are referred to differences between STZ-treated and normal mice, TCVtreated and non-treated mice, respectively (Student's $t$-test). A mouse is considered diabetic when blood glucose level is $\geq$ $300 \mathrm{mg} / \mathrm{dl}$ for 2 consecutive weeks. At least 10 mice were included in each experimental group. Data are representative of three independent experiments.

IPL and levels of TNF- $\alpha$ in the supernatant of SMC. By contrast, TCV treatment increased the levels of both IL-4 and IL-10, whereas it decreased the levels of IL-17 in the culture supernatant of IPL but not in the supernatant of SMC. TCV treatment also decreased the levels of IL23 in the culture supernatants of both intrapancreatic infiltrating cells and SMC. These data indicate that TCV not only shifted the cytokine balance from a Th1 pattern toward the anti-inflammatory $\mathrm{Th} 2$ response as previously reported but also reduced the recently discovered inflam- matory Th17 response in CD4+ T-cell population.

TCV-mediated suppression of IL-17 inflammatory cells through IL-6/Stat 3 signaling pathway

IL-17 has emerged as a crucial regulator of inflammatory responses and is an important cytokine in inflammation and autoimmune disease [27-30]. To better understand the mechanism underlying Th17 suppression, we first measured the IL-17 expression in IPL and SMC from TCV-treated, non-treated MLD-STZ diabetic mice, 

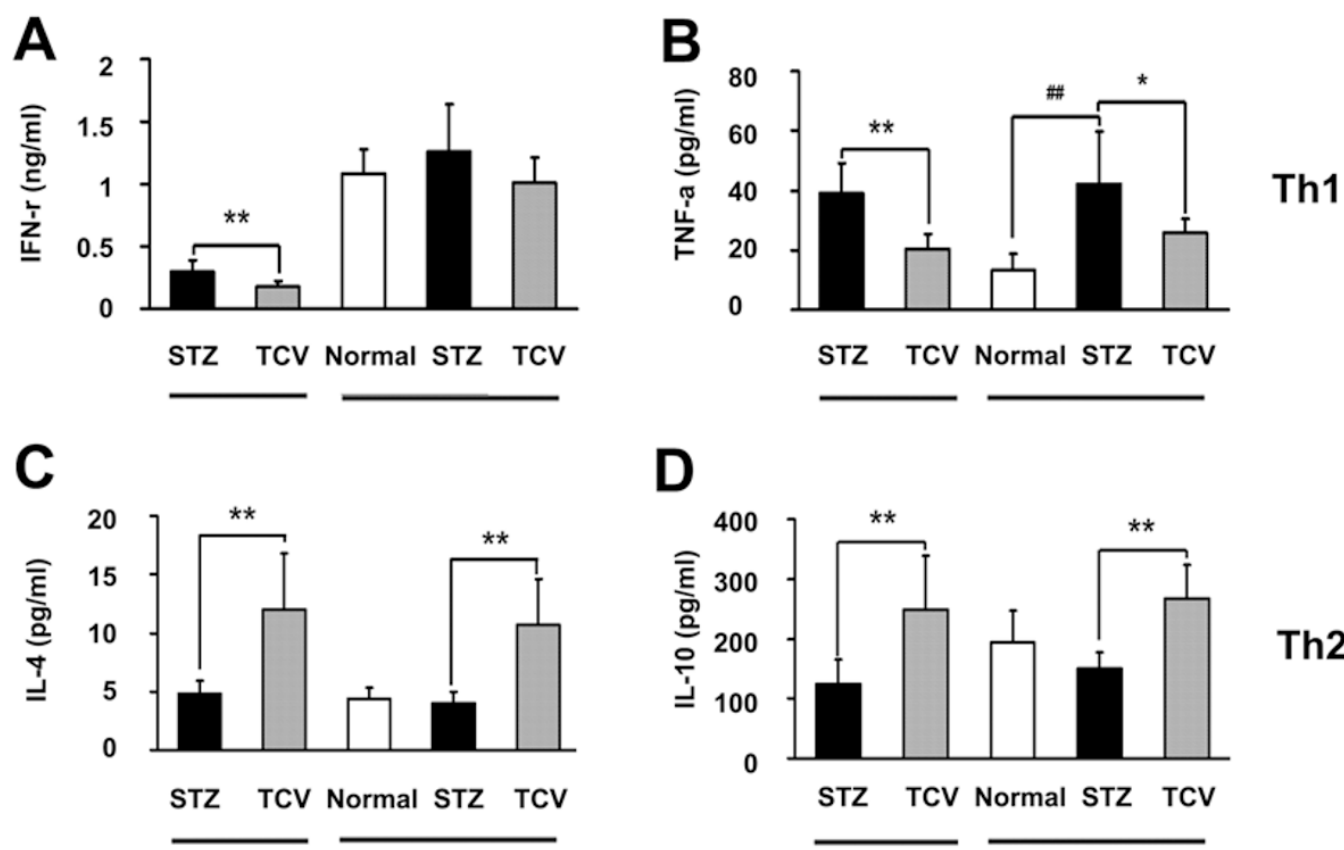

Th2
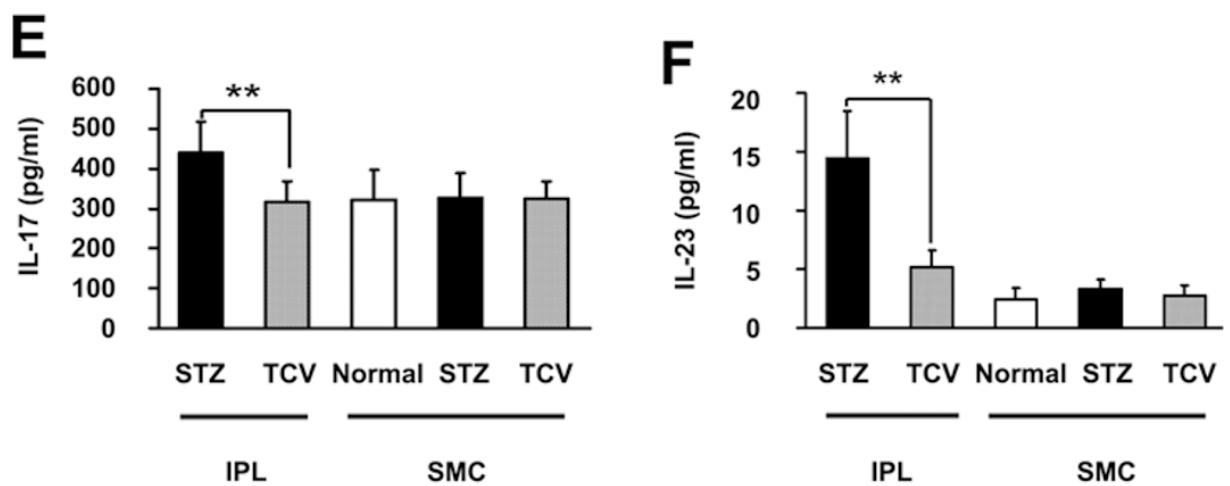

Figure 2 Cytokine profile of intrapancreatic infiltrating lymphocytes (IPL) and spleen mononuclear cells (SMC) in TCV-treated (TCV), non-treated (STZ) MLD-STZ diabetic mice and healthy C57BL/6 mice (normal). The cells were incubated with $5 \mu \mathrm{g} / \mathrm{ml}$ of anti-CD3 antibody for $48 \mathrm{~h}$, and cytokines in the supernatants were measured by ELISA. (A, B) Th1 cytokines, (C, D) Th2 cytokines, (E, F) Th17 cytokines. Each bar represents the mean \pm SD of three independent experiments.

and normal C57BL mice. TCV markedly reduced the frequency of IL-17-producing cells in IPL compared with that in IPL from untreated MLD-STZ diabetic mice (Figure $3 \mathrm{~A}$ and $3 \mathrm{~B}$ ), and significantly decreased the mRNA expression level of IL-17 in intrapancreatic infiltrating lymphoctes but not in SMC (Figure 3C and 3D). Further study revealed that the mRNA level of ROR $\gamma \mathrm{t}$, the Th17 transcription factor, was downregulated in IPL by TCV treatment (Figure 3E and 3F).

Since IL-6 is involved in the polarization of Th-17 and Stat 3 activation is one of the prominent features of IL-6 stimulation, we also investigated the changes of the IL-6/ Stat3 signaling pathway in TCV-treated diabetic mice. Although the production of TGF- $\beta$ showed no differ- ences between the groups, the protein level of IL- 6 was markedly decreased in intrapancreatic infiltrating cells but not in SMC of TCV-treated mice (Figure 4A and 4B). Furthermore, western blot analysis of intrapancreatic infiltrating cells isolated from TCV-treated mice revealed that the phosphorylation of Stat3 (p-Stat3) was also decreased by TCV treatment (Figure 4C and 4D). These findings suggest that TCV inhibited Th17 cells possibly through the IL-6/Stat3 pathway.

TCV decreased blood glucose levels and protected against body weight loss in sub-MLD-STZ-Th17 adoptive transfer diabetic model

To clarify the role of the Th17 cells in the protective 
effect of TCV against autoimmune disease, we used a sub-MLD-STZ-Th17 adoptive transfer diabetic model to further text the effect of TCV. Naive CD4+ T cells derived from 6-week-old C57BL/6 mice were differentiated into Th17 cells under Th17 polarizing condition. The resulting Th17-polarized cells were used for adop-

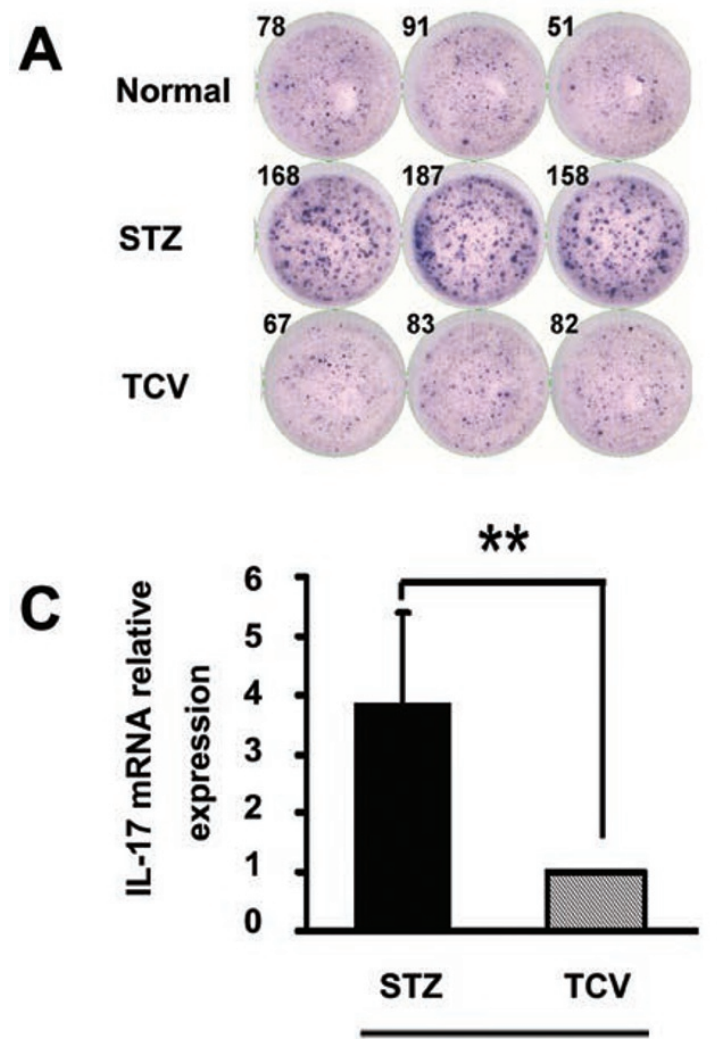

B
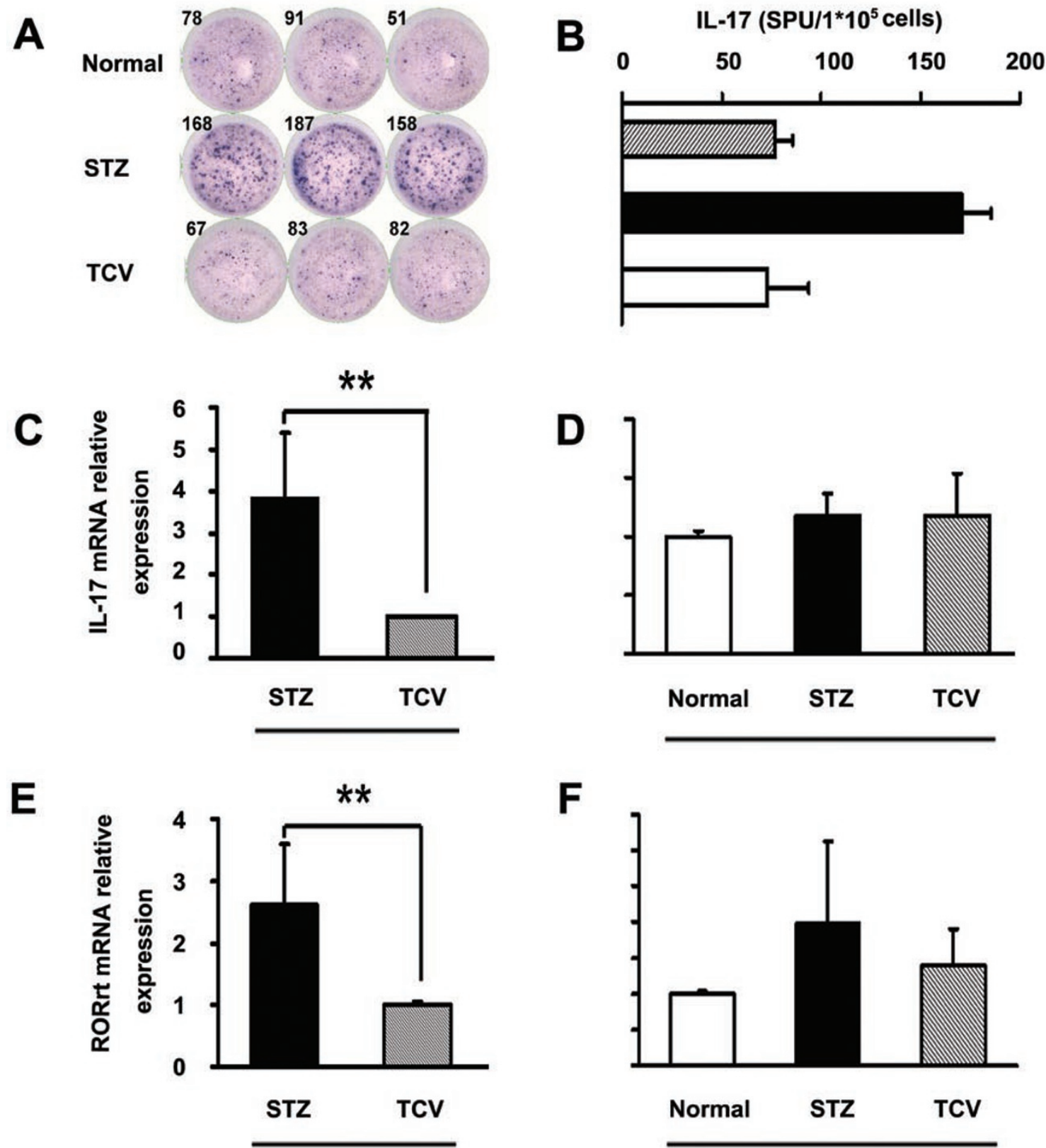

IPL

\section{SMC}

Figure 3 IL-17 expression in intrapancreatic infiltrating lymphocytes (IPL) and spleen mononuclear cells (SMC) in TCVtreated (TCV), non-treated (STZ) MLD-STZ diabetic mice and healthy C57BL/6 mice (normal). (A) The frequencies of IL-17producing cells in intrapancreatic infiltrating lymphocytes were assessed by ELISPOT after the activation with anti-CD3 for $24 \mathrm{~h}$. (B) The number of IL-17 producing cells from (A). (C, D) CD4+ T cells isolated from intrapancreatic infiltrating cells (IPL) and SMC were activated with $5 \mu \mathrm{g} / \mathrm{ml}$ anti-CD3 antibody for $24 \mathrm{~h}$, and IL-17, ROR $\gamma \mathrm{t}$ mRNA expression was determined by realtime PCR. The data shown were normalized to the expression of internal reference gene $\beta$-actin, and the expression level in CD4+ $\mathrm{T}$ cell from healthy C57BL/6 mice was referred as 1 . At least 10 mice were included in each experimental group. Data are representative of three independent experiments. 

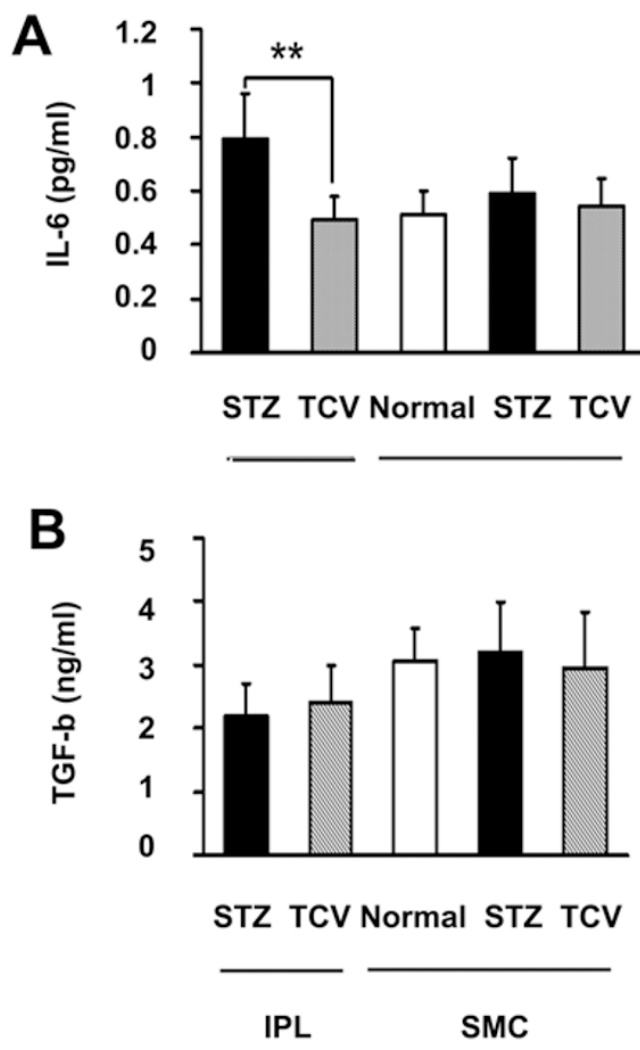
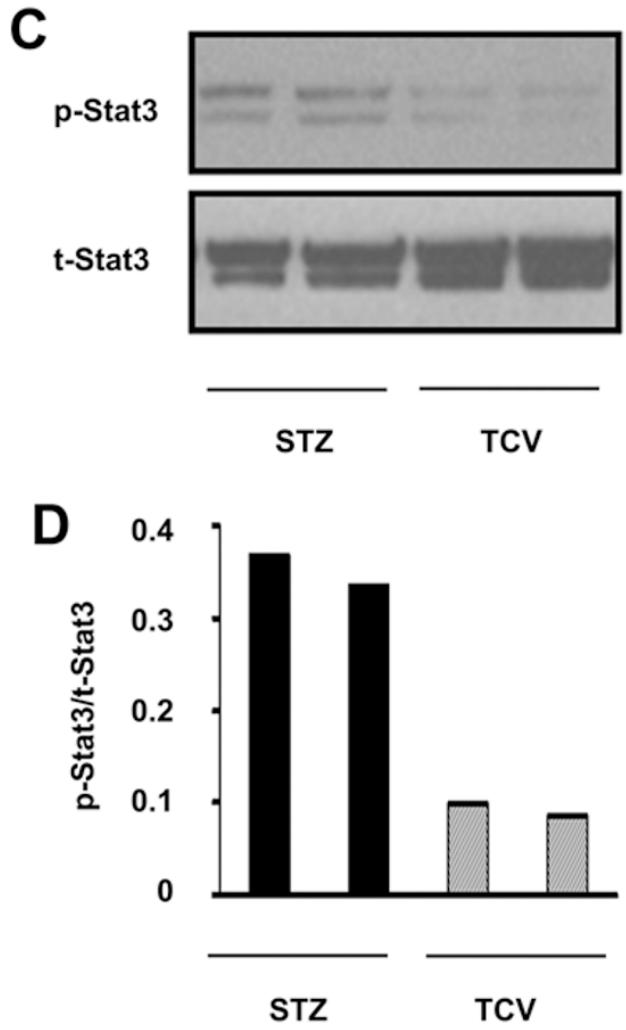

Figure 4 Increased IL-6/Stat3 signaling was suppressed by TCV in intrapancreatic infiltrating lymphocytes in MLD-STZ diabetic mice. (A, B) IL-6 and TGF- $\beta$ expression levels in the supernatant of IPL and SMC after stimulation in vitro with $5 \mu \mathrm{g} / \mathrm{ml}$ anti-CD3 antibody for $48 \mathrm{~h}$ were detected by ELISA. (C) West blotting for p-Stat3/t-Stat3 in IPL on day 21 (D) Quantification of the ratio of $p$-Stat3 to $t-S t a t 3$. Each bar represents the mean \pm SD of three independent experiments.

tive transfer study in sub-MLD-STZ-induced prediabetic mice. The Th17-polarized cells showed an elevated IL17 expression level compared with nonpolarized ones and the recipient mice transferred with the polarized cells manifested diabetes within 16 days, whereas the recipient mice transferred with nonpolarized cells did not (Figure 5A and 5B). The STZ-treated group (STZ), in which mice were injected with STZ at a dose of $40 \mathrm{mg} / \mathrm{kg}$ for 5 consecutive days, developed a sustained high blood glucose level (Figure 5B). The sub-STZ-treated group (subSTZ), in which mice were injected with STZ at a dose of $40 \mathrm{mg} / \mathrm{kg}$ for 4 consecutive days, did not develop a high blood glucose level. The sub-STZ group with adoptive transfer of $10 \times 10^{6} \mathrm{Th} 17$-polarized cells on the first day of the STZ treatment (sub-STZ/Th17) developed a sustained high blood glucose level, similar to the STZtreated group (Figure 5B and 5C). These results indicate that transfer of the Th17-polarized cells enhanced the development of a high blood glucose level and induced evident diabetes in sub-STZ-induced prediabetic mice. However, TCV treatment in this model (sub-STZ/Th17/
TCV) significantly decreased the blood glucose level, which was similar to the effect of neutralization of IL17 by administration of anti-IL-17 antibody (sub-STZ/ Th17/anti-IL17), showing the effect of TCV against the progression of autoimmune diabetes induced by adoptive transfer of Th17 cells in the recipient mice (Figure 5C). Administration of anti-IFN- $\gamma$ (sub-STZ/Th17/anti-IFN $\gamma$ ) or isotype control antibody (sub-STZ/Th17/IgG) did not prevent the development of diabetes in sub-STZ-Th17 adoptive transfer diabetic recipients (Figure 5C). These results further validate the role of Th17-cell suppression in the protective effect of TCV against autoimmune diabetes.

The body weight of TCV-treated mice was monitored. Among the groups monitored (sub-STZ, sub-STZ/Th17, sub-STZ/Th17/TCV, and sub-STZ/Th17/anti-IL-17), the group of sub-STZ/Th17 exhibited body weight loss at each time point of the 35-day measurement period, while TCV completely prevented the weight loss (Figure 5D). Thus, TCV treatment alleviated the weight loss in the sub-MLD-STZ-Th17 adoptive transfer diabetic model, 

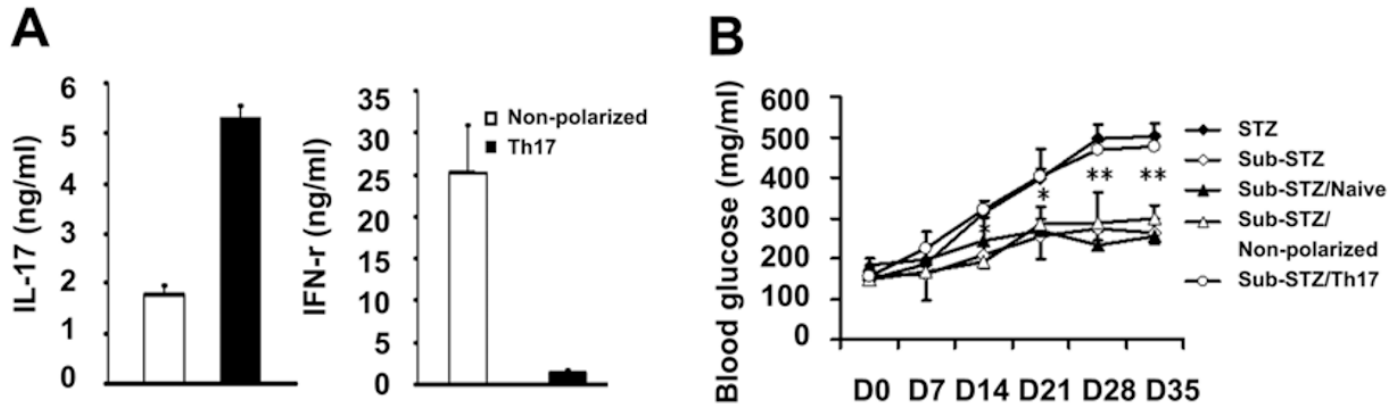

Days post the first STZ injection

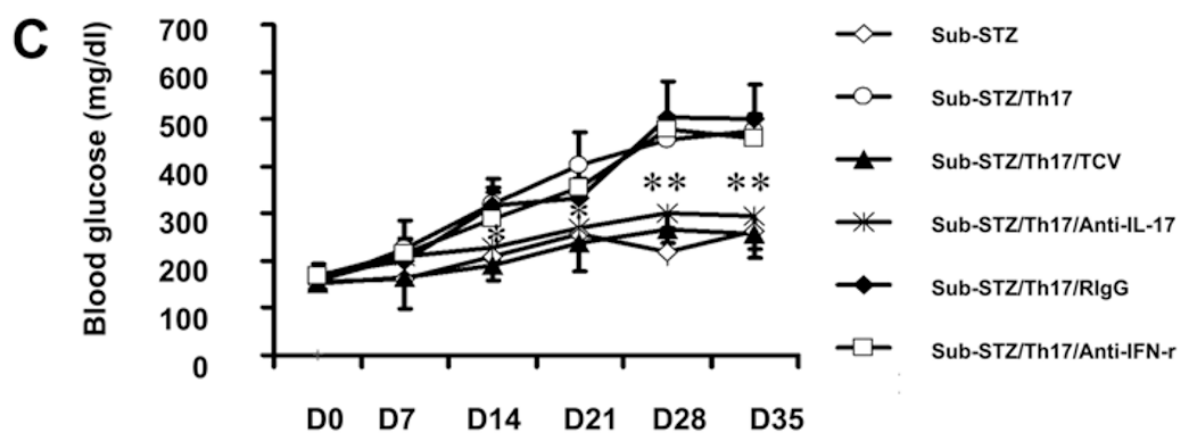

Days post the first STZ injection

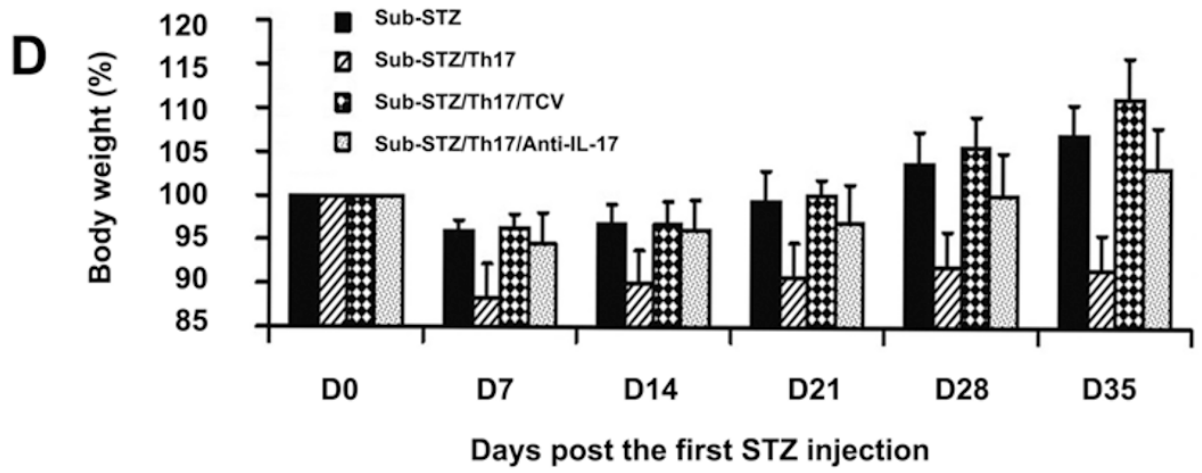

Figure 5 TCV decreased blood glucose levels and protected body weight lost in sub-MLD-STZ/Th17 adoptive transfer diabetic model. (A) IL-17 (left) and INF- $\gamma$ (right) responses from the nonpolarized and Th17 polarized splenocytes were detected by ELISA. Each bar represents the mean \pm SD of triplicate wells. (B) Th17-polarized cells induce diabetes in sub-STZinduced prediabetic mice. Mice were injected with $40 \mathrm{mg} / \mathrm{kg} /$ day STZ for 4 consecutive days (sub-STZ) or 5 (STZ) consecutive days as controls together with adoptive transfer of $10 \times 10^{6}$ Th17-polarized cells (sub-STZ/Th17) on the first day of the STZ regimen. In addition, two groups (sub-STZ/naive, sub-STZ/nonpolarized) received naive spleenocytes or nonpolarized cells, respectively, for control purpose. At least 10 mice were included in each experimental group. Data are representative of three independent experiments. $* P<0.05$; $* P<0.01$, sub-STZ/Th17 vs sub-STZ. (C) Blood glucose levels and (D) percentage weight gain or lost in sub-STZ/Th17 adoptive transfer diabetic mouse model. Mice were injected with $40 \mathrm{mg} / \mathrm{kg} / \mathrm{day}$ STZ for 4 consecutive days (sub-STZ) together with adoptive transfer of $10 \times 10^{6}$ Th17-polarized cells (sub-STZ/Th17) on the first day of the STZ regimen. TCV-treated group was received the total of three TCV inoculations on day 0, 7, 14 (sub-STZ/Th17/ TCV). Additional three groups (sub-STZ/Th17/anti-IL-17, sub-STZ/Th17/anti-IFN- $\gamma$ and sub-STZ/Th17/RIgG) received IL-17 or IFN- $\gamma$-neutralizing antibody or rat IgG, respectively, for control purposes. Anti-IL-17 antibody $(200 \mu \mathrm{g} / \mathrm{mouse})$, anti-INF- $\gamma(500$ $\mu \mathrm{g} /$ mouse $)$ and rat $\operatorname{lgG}(200 \mu \mathrm{g} / \mathrm{mouse})$ were given on the day of transfer, and three additional injections were given on day 4, 7, 14 after transfer. $* P<0.05 ; * * P<0.01$, sub-STZ/Th17/TCV vs sub-STZ/Th17. At least 10 mice were included in each experimental group. Data are representative of three independent experiments. 
which was similar to the effect of anti-IL-17 antibody treatment.

\section{Discussion}

T1D mellitus is an autoimmune disease caused by accumulation of noxious processes of autoimmunity composed of various components of the innate and adaptive immune systems. Auto-reactive T cells directed against one or more $\beta$-cell autoantigens are believed to be involved in the autoimmune process of T1D [1, 31, 32]. Sequential spreading seems to orchestrate T1D, with insulin being required for the initiation of the disease [33], whereas GAD-reactive $\mathrm{T}$ lymphocytes are more involved at later stages of T1D $[34,35]$. Thus, for an antigen-specific therapy to be effective and practical against T1D, it would have to target late-stage epitopes that could counter diverse aggressive T-cell specificities. Oral administration of Escherichia coli GAD has immunomodulatory effects in STZ-induced diabetes and GAD2 peptide corresponding to amino acid sequence 206-220 of GAD was considered a late-stage epitope because its T-cell reactivity was detected at an advanced stage of the disease [36]. A peptide of HSP60 (p277) was found to be useful as a therapeutic agent to arrest the autoimmune process responsible for diabetes in NOD mice and is now in phase II clinical trial [37]. TCV approach targets the immune system by inhibiting the progression of the inflammatory process. This immune-modulating approach attempts to not only cure but also prevent the onset of T1D and thus halt further destruction of the $\beta$-islet cells to avoid the consequences of the endocrine disease.

Concerning the underlying mechanism of TCV, it is believed that TCV activates Th2 type of anti-inflammatory cells, and thus blocks the action of Th1 autoimmune $\mathrm{T}$ cells and switches the autoimmune response to a Th2 phenotype [2]. In other words, Th1 regulators convert a Th1 autoimmune attack into a suppressive Th2 response. In this study, we confirmed that TCV decreased Th1 cytokines such as IFN- $\gamma$ and TNF- $\alpha$ and increased Th 2 cytokines such as IL-4 and IL-10. However, it is not clear whether other T-cell types are also involved in the action of TCV. We hypothesized that the recently discovered Th17 may be involved in the pathogenesis of T1D, since several studies reported the important role of Th17 in autoimmune diseases. Th17 cells are characterized by their production of the cytokine IL-17 [28]. The role of Th17 cells in mediating autoimmune pathology is also increasingly recognized. There are various factors regulating the Th17 development and differentiation. Among them, IL23 is particularly important. This cytokine regulates IL17 production and promotes the expansion of Th17 cells
[38]. IL-23 is considered to be a major driving force in the induction of autoimmune inflammation. Recent study [39] has shown that in an animal model IL-23 is involved in the development of T1D, by inducing IL-17 and possibly IFN- $\gamma$ production in the target tissues.

Interestingly, a decreased Th1 response and an increased Th2 response were observed in both IPL and SMC, whereas the inhibition of Th17 response appeared only in IPL but not in SMC after TCV treatment. STZ dramatically increased the frequency of IL-17-producing cells while TCV decreased the frequency, especially in intrapancreatic infiltrating lymphocytes. By measuring the IL-17 mRNA expression, we found that TCV treatment also decreased IL-17 mRNA expression in IPL but not in spleen mononuclear cells. These results indicate that Th17 cells may play an important role in the inhibitory effect of TCV against autoimmune diabetes. To provide further supporting evidence, we evaluated the mRNA expression of ROR $\gamma \mathrm{t}$. ROR $\gamma \mathrm{t}$ is an orphan nuclear receptor and is selectively expressed in in vitro-differentiated Th17 cells and in IL- $17^{+} \mathrm{T}$ cells present in the lamina propria of naive mice $[40,41]$. ROR $\gamma \mathrm{t}$ is required for IL-17 production, as mice re-constituted with the bone marrow of ROR $\gamma \mathrm{t}$-deficient mice show an impaired Th17 differentiation $[40,41]$. Furthermore, transfection of native $\mathrm{T}$ cells with a retroviral vector expressing ROR $\gamma \mathrm{t}$ induces the development of IL-17-producing Th17 cells [40, 41]. Thus the measurement of ROR $\gamma t$ mRNA expression can be considered as an important parameter to study the role of Th17. The inhibition of ROR $\gamma \mathrm{t}$ mRNA expression by TCV treatment provided further support to the role of Th17 in TCV-induced protection against autoimmune diabetes. Administration of anti-IL-17 antibody effectively prevented the development of diabetes but not antiIFN $\gamma$ or control antibodies. Collectively, these findings indicate that Th17 cells may be directly involved in the inflammatory process of the pancreatic islets, and suppression of Th17 cells in pancreas plays a critical part in TCV-mediated immune responses against STZ-induced diabetes.

To further clarify the role of Th17 in the TCV-mediated protection against T1D, we examined the levels of IL- 6 and TGF- $\beta$. IL- 6 is a multifunctional cytokine that regulates immune response, hematopoiesis, acute phase response, and inflammation [38]. IL-6 inhibits the function of regulatory $\mathrm{T}$ cells and promotes the differentiation of Th17 cells [42], and IL-6-deficient mice lack the ability to generate Th17 cells [43]. This cytokine together with TGF- $\beta$ is a strong inducer of Th-17. The results obtained from the present study showed that TCV decreased the production of IL- 6 but not TGF- $\beta$ in intrapancreatic infiltrating cells. IL-6 acts directly to promote 
the development of Th17 by activating the T-cell gp130Stat3 pathway $[44,45]$. Inhibition of Stat 3 and its related Th17 development is an important approach to mitigating pathogenic immunity. Thus, we also examined the phosphorylation status of Stat 3 after TCV treatment and our results showed decreased p-Stat 3 as expected. Taken together, these results indicate that TCV treatment can reduce the production of IL-6, hence to inhibit Stat3 activation and Th17 development.

Another important finding to support the role of Th17 cells in the protective effect of TCV against autoimmune disease in T1D is the measurement of the blood glucose level and body weight in the sub-MLD-STZTh17 adoptive transfer diabetic animal model. It has been established that five daily doses of $40 \mathrm{mg} / \mathrm{kg} /$ day are required for delayed onset, sustained and progressive hyperglycemia and insulitis in male C57BL/6 mice and the 4-day injection of STZ did not induce significant hyperlycemia and only led to mild single cell insulitis [39]. The following results collectively support our working hypothesis that TCV exhibits its function against autoimmune T1D via the suppression of Th17 cells. First, the sub-STZ-treated animals in which mice were injected with STZ at a dose of $40 \mathrm{mg} / \mathrm{kg}$ for 4 consecutive days, did not develop a high blood glucose level. These subSTZ-treated animals with adoptive transfer of $10 \times 10^{6}$ Th17-polarized cells on the first day of the STZ treatment developed a sustained high blood glucose level, similar to the STZ-treated group, in which mice were injected with STZ at a dose of $40 \mathrm{mg} / \mathrm{kg}$ for 5 consecutive days. Second, TCV treatment of sub-STZ-treated animals that received the adoptive transfer of $10 \times 10^{6}$ Th17-polarized cells decreased the blood glucose level, showing the effectiveness of TCV against the progression of autoimmune diabetes induced by STZ. Third, the anti-IL-17 antibody treatment of sub-STZ-treated animals that received the adoptive transfer of $10 \times 10^{6}$ Th17-polarized cells decreased the blood glucose level, showing that the suppression of IL-17 protects against the progression of autoimmune diabetes induced by STZ. Previous study showed that TCV exerts immunomodulatory effect through the induction of both anti-idiotypic response, which was specific to the immunizing cells and anti-ergotypic $\mathrm{T}$ cells more toward activated $\mathrm{T}$ cells in other autoimmune diseases [46-49]. It is not unlikely that suppression of Th17 cells is the result of TCV-induced anti-ergotypic effect because Th17 cells are an activated T-cell subset. Whether the TCV treatment-induced Thl/ Th2 shift and Th17 suppression in STZ-induced diabetes involve anti-ergotypic mechanism awaits further investigation.

In summary, TCV inhibited the development of auto- immune diabetes induced by multiple low doses of STZ through suppression of Th17 cells. Over the years, the Th1/Th2 paradigm of T-helper cell differentiation has helped to explain many phenomena in adaptive immunity. Recently, the Th1/Th2 paradigm has been expanded, following the discovery of a third subset of the effector T-helper cells that produce IL-17 and exhibit effector functions distinct from Th1 and Th2 cells. Th17 cells are potent inducers of tissue inflammation and have been associated with the pathogenesis of many experimental autoimmune diseases and human inflammatory conditions. This study demonstrated that Th17 cells are also important in MLD-STZ-induced T1D, and TCV-mediated suppression of Th17 cells played a critical role in its protective effect against autoimmune diabetes induced by MLD-STZ.

\section{Materials and Methods}

\section{Mice}

Male C57BL/6J inbred mice (6-8 weeks of age) were purchased from Shanghai Slac Laboratory Animal Co. Ltd (Shanghai, China). All mice were maintained in a temperature-controlled $\left(23{ }^{\circ} \mathrm{C}\right)$ facility with a 12 -h light/dark cycle and were given free access to food and water. All animal experiments were repeated. All experiments were performed following the Shanghai Institute for Biological Sciences of the Chinese Academy of Sciences for animal studies.

\section{Induction of MLD-STZ diabetic mice and sub-diabetogenic mice}

Autoimmune diabetes was induced using multiple low doses of STZ (Sigma-Aldrich, St Louis, MO, USA) as described in Leiter [50]. Briefly, male mice (6-8 weeks of age) were injected i.p. with STZ (40 mg/kg freshly dissolved in cold $0.1 \mathrm{M}$ citrate buffer $(\mathrm{pH}$ 4.5) for 4 (sub-MLD-STZ diabetic mice) or 5 (MLD-STZ diabetic mice) consecutive days. Blood was obtained from the tail vein of non-fasted mice and blood glucose level was monitored twice a week over the following 56 days using an Ascensia Elite XL onetouch blood glucometer (Bayer, NJ, USA). Mice were considered to be diabetic if the blood glucose levels were beyond $300 \mathrm{mg} / \mathrm{dl}$ in two consecutive daily measurements. Mice in the control group received an equal volume of sodium citrate buffer alone. At least 10 mice were included in each experimental group.

\section{Peptides}

GAD65 peptide p206-220 (TYEIAPVFVLLEYVT) and HEL peptide encompasses a non-diabetogenic epitope corresponding to aa residues 11-25 (AMKRHGLDNYRGYSL) of HEL were synthesized by GL Biomedicals (Shanghai, China) using an automated peptide synthesis system (Pioneer: PerSeptive Biosystems, Framingham, MA, USA). The crude peptide was purified by reversed-phase HPLC using a $\mathrm{C} 18$ column with a gradient of solvent A $\left(95 \% / 5 \%=\mathrm{H}_{2} \mathrm{O}(0.1 \%\right.$ TFA $) /$ acetonitrile $)$ and solvent B $(100 \%$ acetonitrile). The purity of the peptide was analyzed by analytical HPLC using an analytical C18 column and showed 95\% purity. The identity of the synthesized peptide was confirmed by matrix- 
assisted laser desorption/ionization time-of-flight mass spectrometry.

\section{Animal immunization and generation of peptide-specific short-term T-cell lines}

Male C57BL/6J mice (6-8 weeks of age) were immunized in both hind footpads with $200 \mu \mathrm{g}$ of peptide, respectively, emulsified in IFA (Sigma-Aldrich). At 10 days later, immunized mice were sacrificed and SMCs were prepared from the spleens of GAD65 peptide p206-220-immunized mice and HEL peptide p1125 -immunized mice and plated out at $10^{7}$ cells/well in 24-well culture plates in the presence of $20 \mu \mathrm{g} / \mathrm{ml}$ the peptide, respectively [51]. After 7 days of incubation, all cultures were re-stimulated with irradiated autologous SMC (3 $000 \mathrm{rad}$, Co60) pulsed with the peptide, and supplemented with rIL-2 at $100 \mathrm{IU} / \mathrm{ml}$ after $48 \mathrm{~h}$. After another 7 days, all cultures were examined for specific proliferation in response to the peptide using ${ }^{3} \mathrm{H}$ proliferation assays. Briefly, each culture was collected and re-plated at $10^{5} \mathrm{cell} /$ well in 96-well round bottom plates in duplicates with irradiated $10^{5}$ autologous SMC in the presence or absence of the peptide. Cultures were kept for 3 days and pulsed with $\left[{ }^{3} \mathrm{H}\right]$ thymidine (Amersham, Braunschweig, Germany) at $1 \mu \mathrm{Ci}$ per well during the last $16 \mathrm{~h}$ of culture. Cells were then harvested using an automated cell harvester and $\left[{ }^{3} \mathrm{H}\right]$ thymidine incorporation was measured in a betaplate counter (Wallac, Turku, Finland). A well/culture was defined as specific for antigen peptide when the c.p.m. was greater than 1500 and exceeded the reference c.p.m. (in the absence of antigen peptide) by at least three times.

\section{TCV protocol}

GAD65 peptide p206-220-specific T-cell line and HEL peptide p11-25-secific T-cell line were pre-activated with $2 \mu \mathrm{g} / \mathrm{ml}$ Con-A (Sigma-Aldrich) in the presence of irradiated SMC as feeder cells. Cells were cultured for 5-6 days in RPMI1640 media supplemented with $10 \%$ heat-inactivated autologous serum and $50 \mathrm{U}$ of rIL2. Activated peptide-specific $\mathrm{T}$ cells were subsequently washed three times with sterile saline. After irradiation (3 $000 \mathrm{rad}, 60 \mathrm{Co}$ source), cells were washed and re-suspended in sterile saline. Each MLD-STZ diabetic mouse received a total of three TCV injections given s.c. in both hind footpads at 7-day intervals. The number of $\mathrm{T}$ cells used for immunization ranged from $5 \times 10^{4}$ to $10 \times 10^{4}$ cells per injection. The TCV treatment was begun at the same time the mice received the first dose of STZ and STZ was given first followed by TCV.

\section{Histology and immunohistochemistry}

Pancreas were harvested from TCV-treated MLD-STZ diabetic, non-treated MLD-STZ diabetic, and normal control mice, respectively, and fixed in $10 \%$ formalin, and embedded in paraffin. Sections of $8 \mu \mathrm{m}$ thickness were cut $100 \mu \mathrm{m}$ apart to prevent double counting of the same islets. Six sections per pancreas were stained with hematoxylin and eosin and analyzed by light microscopy. Insulitis scoring was performed according to the following criteria: severe insulitis, $50 \%$ or higher of the islet area is infiltrated; mild insulitis, $<50 \%$ of the islet area is infiltrated; periinsulitis, infiltration is restricted to the periphery of islets; and no insulitis, absence of cell infiltration [52]. For assessment of insulin production in pancreas, the sections were stained with primary rat anti-insulin antibodies, incubated with biotinylated goat anti-rat antibodies, and visualized by saturation with streptavidin alkaline phosphatase using the chromagen, 5-bromo-4-chloro-3-indolyl phosphate/nitroblue tetrazolium.

\section{Isolation of intrapanceatic infiltrating lymphocytes}

Intrapanceatic infiltrating lymphocytes from mice were isolated as previously described [53]. Briefly, the pancreas was digested with collagenase type IV (Invitrogen, CA, USA), and islets were separated on a ficoll gradient (GE Healthcare, Little Chalfont, UK).

\section{Measurements of cytokine production}

Culture supernatants of IPL and SMC from TCV treated, nontreated MLD-STZ diabetic and control mice were collected and diluted for the measurements of cytokine concentration using ELISA kits according to the manufacturer's instruction (R\&D Systems, Minneapolis, MN, USA).

\section{Enzyme-linked immunosorbent spot (ELISPOT) assay}

The ELISPOT assays were performed using an IL-17 ELISPOT set (R\&D System). Cells were seeded out at $1 \times 10^{5}$ per well in triplicate wells in the presence of rIL-2 $(40 \mathrm{U} / \mathrm{ml})$ and rIL-23 (10 $\mathrm{ng} / \mathrm{ml}$; R\&D System). After $24 \mathrm{~h}$, cells were restimulated with 500 $\mathrm{ng} / \mathrm{ml}$ ionomycin and $50 \mathrm{ng} / \mathrm{ml}$ phorbol 12-myristate 13 -acetate (Sigma-Aldrich) in the presence of Golgi-stop for $5 \mathrm{~h}$, after which the plates were washed and a biotinylated detection antibody was added and incubated for $12 \mathrm{~h}$ at $4{ }^{\circ} \mathrm{C}$. After another washing step, streptavidin-horseradish peroxidase was added and incubated for $2 \mathrm{~h}$ at room temperature. Spots were developed using AEC solution (Pierce Pharmaceuticals, Rockford, IL, USA) for 10-20 min and the plates/membranes were then washed and air-dried. The image analysis was performed using a Series 1 ImmunoSpot Image Analyzer (Cellular Technology, Cleveland, OH, USA). Digitized images were analyzed and results were expressed as number of positive spots per total number of cells per well. The final frequency was calculated using Poisson statistics.

\section{Western blotting}

To detect the ratio of $\mathrm{p}$-Stat3/t-Stat3 in IPL from TCV-treated and non-treated MLD-STZ diabetic mice, $60 \mu \mathrm{g}$ of total protein from respective cell lysates were subjected to SDS polyacrylamide gel electrophoresis and transferred to PVDF membrane. Membranes were blotted with phospho-specific Stat 3 antibody (Tyrosine 705; \#9131 Cell Signaling Tech, Beverly, MA, USA), which does not cross react with any other Stat protein.

\section{T-cell polarization and adoptive transfer experiments}

Naive splenocytes were isolated from male 6-week-old C57BL/6 mice and activated with soluble anti-CD3 $(5 \mu \mathrm{g} / \mathrm{ml})$ and anti-CD28 $(5 \mu \mathrm{g} / \mathrm{ml})$ antibodies for 3 days in $10 \%$ FCS-DME media under Th17 polarizing mixture consisting of TGF- $\beta(5 \mathrm{ng} / \mathrm{ml})$, IL-6 $(20 \mathrm{ng} / \mathrm{ml})$, anti-IFN- $\gamma$ antibody $(10 \mu \mathrm{g} / \mathrm{ml})$, and anti-IL-4 antibody $(10 \mu \mathrm{g} / \mathrm{ml})$. Supernatant from activated cells was tested for IFN- $\gamma$ and IL-17 by ELISA, and the cells were used for adoptive transfers. For adoptive transfer by Th17 cells, $10 \times 10^{6}$ Th17polarized cells were injected i.v. into sub-MLD-STZ diabetic mice on the first day of the STZ injection.

\section{Statistical analysis}

Data are expressed as means $\pm \mathrm{SD}$. An unpaired $t$-test, one-way ANOVA, and repeated measures ANOVA were used to determine 
statistical significance. Differences were considered significant when $P<0.05$.

\section{Acknowledgments}

We thank Jiankang Liu (University of Kentucky) for critical reading of the manuscript. This work was supported in part by the Chinese Academy of Sciences (KSCX2-YW-G-057) and the Ministry of Science and Technology of China (2007CB914801 and 2009CB919000).

\section{References}

1 Foulis AK, Stewart JA. The pancreasss in recent-onset type 1 (insulin-dependent) diabetes mellitus: insulin content of islets, insulitis and associated changes in the exocrine acinar tissue. Diabetologia 1984; 26:456-461.

2 Cohen IR. T-cell vaccination for autoimmune disease: a panorama. Vaccine 2001; 20:706-710.

3 Waisman A, Ruiz PJ, Hirschberg DL, et al. Suppressive vaccination with DNA encoding a variable region gene of the T-cell receptor prevents autoimmune encephalomyelitis and activates Th2 immunity. Nat Med 1996; 2:899-905.

4 Elias D, Tikochinski Y, Frankel G, Cohen IR. Regulation of NOD mouse autoimmune diabetes by $\mathrm{T}$ cells that recognize a TCR CDR3 peptide. Int Immunol 1999; 11:957-966.

5 Kumar V, Sercarz E. Induction or protection from experimental autoimmune encephalomyelitis depends on the cytokine secretion profile of TCR peptide-specific regulatory CD4 T cells. J Immunol 1998; 161:6585-6591.

6 Vermeire K, Heremans H, Vandeputte M, Huang S, Billiau A, Matthys P. Accelerated collagen-induced arthritis in IFNgamma receptor-deficient mice. J Immunol 1997; 158:55075513.

7 Murphy CA, Langrish CL, Chen Y, et al. Divergent pro- and antiinflammatory roles for IL-23 and IL-12 in joint autoimmune inflammation. J Exp Med 2003; 198:1951-1957.

8 Chu CQ, Wittmer S, Dalton DK. Failure to suppress the expansion of the activated CD4 T cell population in interferon gamma-deficient mice leads to exacerbation of experimental autoimmune encephalomyelitis. J Exp Med 2000; 192:123128.

9 Hultgren B, Huang X, Dybdal N, Stewart TA. Genetic absence of gamma-interferon delays but does not prevent diabetes in NOD mice. Diabetes 1996; 45:812-817.

10 Manoury-Schwartz B, Chiocchia G, Bessis N, et al. High susceptibility to collagen-induced arthritis in mice lacking IFNgamma receptors. J Immunol 1997; 158:5501-5506.

11 Cua DJ, Sherlock J, Chen Y, et al. Interleukin-23 rather than interleukin-12 is the critical cytokine for autoimmune inflammation of the brain. Nature 2003; 421:744-748.

12 Trembleau S, Penna G, Gregori S, et al. Pancreas-infiltrating Th1 cells and diabetes develop in IL-12-deficient nonobese diabetic mice. J Immunol 1999; 163:2960-2968.

13 McKenzie BS, Kastelein RA, Cua DJ. Understanding the IL23-IL-17 immune pathway. Trends Immunol 2006; 27:17-23.

14 Iwakura Y, Ishigame H. The IL-23/IL-17 axis in inflammation. J Clin Invest 2006; 116:1218-1222.

15 Park H, Li Z, Yang XO, et al. A distinct lineage of CD4 T cells regulates tissue inflammation by producing interleukin 17. Nat Immunol 2005; 6:1133-1141.

16 Langrish CL, Chen Y, Blumenschein WM, et al. IL-23 drives a pathogenic $\mathrm{T}$ cell population that induces autoimmune inflammation. $J$ Exp Med 2005; 201:233-240.

17 Dong G, Ye R, Shi W, et al. IL-17 induces autoantibody overproduction and peripheral blood mononuclear cell overexpression of IL-6 in lupus nephritis patients. Chin Med J (Engl) 2003; 116:543-548.

18 Weiss RB. Streptozocin: a review of its pharmacology, efficacy, and toxicity. Cancer Treat Rep 1982; 66:427-438.

19 Ohly P, Dohle C, Abel J, Seissler J, Gleichmann H. Zinc sulphate induces metallothionein in pancreatic islets of mice and protects against diabetes induced by multiple low doses of streptozotocin. Diabetologia 2000; 43:1020-1030.

20 Friesen NT, Buchau AS, Schott-Ohly P, Lgssiar A, Gleichmann H. Generation of hydrogen peroxide and failure of antioxidative responses in pancreatic islets of male $\mathrm{C} 57 \mathrm{BL} / 6$ mice are associated with diabetes induced by multiple low doses of streptozotocin. Diabetologia 2004; 47:676-685.

21 Choi SE, Noh HL, Kim HM, Yoon JW, Kang Y. Streptozotocin upregulates GAD67 expression in MIN6N8a mouse beta cells. J Autoimmun 2002; 19:1-8.

22 Han G, Li Y, Wang J, et al. Active tolerance induction and prevention of autoimmune diabetes by immunogene therapy using recombinant adenoassociated virus expressing glutamic acid decarboxylase 65 peptide GAD(500-585). J Immunol 2005; 174:4516-4524.

23 Elias D, Prigozin H, Polak N, Rapoport M, Lohse AW, Cohen IR. Autoimmune diabetes induced by the beta-cell toxin STZ. Immunity to the $60-\mathrm{kDa}$ heat shock protein and to insulin. Diabetes 1994; 43:992-998.

24 Jun HS, Chung YH, Han J, et al. Prevention of autoimmune diabetes by immunogene therapy using recombinant vaccinia virus expressing glutamic acid decarboxylase. Diabetologia 2002; 45:668-676.

25 King GL. The role of inflammatory cytokines in diabetes and its complications. J Periodontol 2008; 79(8 Suppl):1527-1534.

26 Goldberg RB. Cytokine and cytokine-like inflammation markers, endothelial dysfunction, and imbalanced coagulation in development of diabetes and its complications. J Clin Endocrinol Metab 2009; 94:3171-3182.

27 Dong C. Diversification of T-helper-cell lineages: finding the family root of IL-17-producing cells. Nat Rev Immunol 2006; 6:329-333.

28 Kramer JM, Gaffen SL. Interleukin-17: a new paradigm in inflammation, autoimmunity, and therapy. J Periodontol 2007; 78:1083-1093.

29 Bettelli E, Oukka M, Kuchroo VK. T(H)-17 cells in the circle of immunity and autoimmunity. Nat Immunol 2007; 8:345350 .

30 Dong C. TH17 cells in development: an updated view of their molecular identity and genetic programming. Nat Rev Immunol 2008; 8:337-348.

31 Tisch R, McDevitt H. Insulin-dependent diabetes mellitus. Cell 1996; 85:291-297.

32 Bach JF. Insulin-dependent diabetes mellitus as an autoimmune disease. Endocr Rev 1994; 15:516-542. 
33 Nakayama M, Abiru N, Moriyama H, et al. Prime role for an insulin epitope in the development of type 1 diabetes in NOD mice. Nature 2005; 435:220-223.

34 Nepom GT, Lippolis JD, White FM, et al. Identification and modulation of a naturally processed $\mathrm{T}$ cell epitope from the diabetes-associated autoantigen human glutamic acid decarboxylase 65 (hGAD65). Proc Natl Acad Sci USA 2001; 98:1763-1768.

35 Kaufman DL, Clare-Salzler M, Tian J, et al. Spontaneous loss of T-cell tolerance to glutamic acid decarboxylase in murine insulin-dependent diabetes. Nature 1993; 366:69-72.

36 Yazdchi-Marandi L, Yazdanparast R, Rafieii S. Oral administration of Escherichia coli glutamic acid decarboxylase has immunomodulatory effects in streptozotocin-induced diabetes. Microbios 2000; 102:135-145.

37 Elias D, Cohen IR. The hsp60 peptide p277 arrests the autoimmune diabetes induced by the toxin streptozotocin. Diabetes $1996 ; \mathbf{4 5}: 1168-1172$.

38 Ishihara K, Hirano T. IL-6 in autoimmune disease and chronic inflammatory proliferative disease. Cytokine Growth Factor Rev 2002; 13:357-368.

39 Mensah-Brown EP, Shahin A, Al-Shamisi M, Wei X, Lukic ML. IL-23 leads to diabetes induction after subdiabetogenic treatment with multiple low doses of streptozotocin. Eur $J$ Immunol 2006; 36:216-223.

40 Korn T, Bettelli E, Oukka M, Kuchroo VK. IL-17 and Th17 cells. Annu Rev Immunol 2009; 27:485-517.

41 Ivanov II, McKenzie BS, Zhou L, et al. The orphan nuclear receptor RORgammat directs the differentiation program of proinflammatory IL-17+ T helper cells. Cell 2006; 126:11211133.

42 Oukka M. Th17 cells in immunity and autoimmunity. Ann Rheum Dis 2008; 67 Suppl 3:iii26-iii29.

43 Egwuagu CE. STAT3 in CD4+ T helper cell differentiation and inflammatory diseases. Cytokine 2009; 47:149-156.
44 Nishihara M, Ogura H, Ueda N, et al. IL-6-gp130-STAT3 in T cells directs the development of IL-17+ Th with a minimum effect on that of Treg in the steady state. Int Immunol 2007; 19:695-702.

45 Lukic ML, Mensah-Brown E, Wei X, Shahin A, Liew FY. Lack of the mediators of innate immunity attenuate the development of autoimmune diabetes in mice. J Autoimmun 2003; 21:239-246.

46 Quintana FJ, Cohen IR. Anti-ergotypic immunoregulation. Scand J Immunol 2006; 64:205-210.

47 Cohen IR, Quintana FJ, Mimran A. Tregs in T cell vaccination: exploring the regulation of regulation. $J$ Clin Invest 2004; 114:1227-1232.

48 Van der Aa A, Hellings N, Medaer R, et al. T cell vaccination in multiple sclerosis patients with autologous CSF-derived activated T cells: results from a pilot study. Clin Exp Immunol 2003; 131:155-168.

49 Hellings N, Raus J, Stinissen P. T-cell vaccination in multiple sclerosis: update on clinical application and mode of action. Autoimmun Rev 2004; 3:267-275.

50 Leiter EH. Multiple low-dose streptozotocin-induced hyperglycemia and insulitis in C57BL mice: influence of inbred background, sex, and thymus. Proc Natl Acad Sci USA 1982; 79:630-634.

51 Quinn A, McInerney MF, Sercarz EE. MHC class I-restricted determinants on the glutamic acid decarboxylase 65 molecule induce spontaneous CTL activity. J Immunol 2001; 167:17481757.

52 Jain R, Tartar DM, Gregg RK, et al. Innocuous IFNgamma induced by adjuvant-free antigen restores normoglycemia in NOD mice through inhibition of IL-17 production. $J$ Exp Med 2008; 205:207-218.

53 Kingsley G, Panayi GS. Management of early inflammatory arthritis. Intervention with immunomodulatory agents: $\mathrm{T}$ cell vaccination. Baillieres Clin Rheumatol 1992; 6:435-454. 